\title{
Aplicación complementaria de terapia
} fotodinámica y de la radiación láser de Er:YAG al tratamiento no quirúrgico de la periodontitis crónica: estudio comparativo de sus efectos clínicos, antiinflamatorios y antimicrobianos

\section{Adjunctive application of the photodynamic therapy and the Er:YAG laser radiation to non-surgical treatment of chronic periodontitis: comparative study of their clinical, anti-inflammatory and antimicrobial effects}

Gómez Hernández C*, Domínguez Martín A*, García Kass Al**, García Núñez JA**

\section{RESUMEN}

Objetivos: Comparar efectos clínicos, antimicrobianos y antiinflamatorios de la terapia fotodinámica (TFD) y de la fototerapia con láser de Er:YAG aplicadas complementariamente al raspado y alisado radicular (RAR). Material y métodos: 45 pacientes con enfermedad periodontal crónica, fueron distribuidos aleatoriamente en 3 grupos de 15 para recibir: RAR, RAR complementado con terapia fotodinámica (RAR+TFD) o complementado con láser de Er:YAG (RAR+ERL). Se analizaron los parámetros clínicos: porcentaje de placa en boca total (PPBT), porcentaje de sangrado en boca total (PSBT) y profundidad de sondaje (PS), y se procedió a la recogida de fluido crevicular gingival (FCG) y de placa subgingival antes del tratamiento, a las 4 y 8 semanas posteriores. Se determinaron los niveles de Interleuquina $1 \beta(\mathrm{IL}-1 \beta)$, Factor de Necrosis Tumoral $\alpha$ (FNT- $\alpha$ ) en FCG y su estado antioxidante total (EAT). Las muestras de flora subgingival fueron cultivadas para la determinación de 10 periodontopatógenos. Resultados y discusión: No se encontraron diferencias estadísticamente significativas (DES) entre los tres tipos de tratamiento en cuanto a la evolución de los parámetros clínicos PPBT, PS, ni en cuanto al aumento de la capacidad antioxidante del FCG tras el tratamiento, ni tampoco en cuanto a la reducción de la flora subgingival total analizada, aunque sí se aprecia una ligera ralentización del proceso de recolonización bacteriana en el grupo RAR+Er:YAG. En cuanto al parámetro clínico PSBT, se observó un mayor descenso a las 8 semanas post-tratamiento en el grupo RAR+TFD, aunque no significativo. Sí se encontraron DES en cuanto a la evolución de los niveles de las citoquinas evaluadas: después del RAR los niveles de IL- $1 \beta$ y FNT- $\alpha$ se incrementaron y significativamente en el caso de FNT- $\alpha$ a las 8 semanas post-tratamiento. El procedimiento RAR+TFD mostró una tendencia a frenar dicho incremento y tras la aplicación de RAR+ERL dichos niveles descendieron, de forma más marcada en el caso de la IL- $1 \beta$, durante el periodo de post-tratamiento evaluado.

Palabras clave: Terapia fotodinámica, Er:YAG, RAR, periodontitis crónica.

\section{SUMMARY}

Aim: To compare clinical, microbiological and anti-inflammatory effects of the photodynamic therapy (PDT) and the Er:YAG laser phototherapy adjunctively applied to scaling and root planning (SRP).

* Departamento Química Láser, Instituto de Química Física Rocasolano, CSIC, 28006 Madrid, España.

Departamento de Estomatología III, Facultad de Odontología, UCM, 28040 Madrid, España. 
Material and Methods: Forty-five patients with chronic periodontitis were randomly assigned into 3 groups of 15 patients to receive: SRP, SRP followed by PDT (SRP+PDT) or SRP followed by Er:YAG laser phototherapy (SRP+ERL). Clinical parameters including full mouth plaque score (FMPS), full mouth bleeding score (FMBS) and probing pocket depth (PPD) were recorded and samples of gingival crevicular fluid (GCF) and subgingival plaque were taken at baseline, 4 and 8 weeks postoperatively. The GCF samples were analyzed for interleukin1 beta (IL-1 $\beta$ ), tumour necrosis factor alpha (TNF- $\alpha$ ) and total antioxidative status (TAS). Subgingival plaque was analyzed by culture for 10 bacteria. Results and Discussion: No statistically significant differences were detected between the three kinds of treatment concerning the evolution of the clinical parameters FMPS and PPD, the increase of TAS in GCF and the subgingival plaque reduction, but the SRP+ERL treatment slightly slowed down the bacterial recolonization process. Concerning the clinical parameter FMBS, a slight decrease was observed 8 weeks post-therapy after SRP+PDT treatment. Statistically significant differences between groups were observed in the evolution of the levels of the cytokines evaluated: after SRP the levels of IL- $1 \beta$ and TNF- $\alpha$ increased, significatively in the case of TNF- $\alpha$ at 8 weeks post-therapy. The procedure SRP+PDT showed a trend to slow down this increase, and after the application of SRP+ERL these levels decreased, more severely in the case of IL- $1 \beta$, during the whole post-therapy period.

Key words: Photodynamic therapy, Er:YAG, SRP, chronic periodontitis.

Fecha de recepción: 15 de noviembre de 2009.

Aceptado para publicación: 11 de enero de 2010.

Gómez Hernández C, Domínguez Martín A, García Kass AI, García Núñez JA. Aplicación complementaria de terapia fotodinámica y de la radiación láser de Er:YAG al tratamiento no quirúrgico de la periodontitis crónica: estudio comparativo de sus efectos clínicos, antiinflamatorios y antimicrobianos. Av. Odontoestomatol 2011; 27 (3): 147-160.

\section{INTRODUCCIÓN}

La eliminación de biofilm y los depósitos mineralizados de la superficie dental es el aspecto fundamental de la terapia periodontal (1). En el tratamiento periodontal, los conceptos actuales se basan en el raspado y alisado radicular llevado a cabo de forma mecánica con el fin de eliminar los depósitos bacterianos, cálculo y cemento contaminado por bacterias y endotoxinas (2). Desafortunadamente, la eficacia del desbridamiento varía en los diferentes casos clínicos por la existencia de reservorios bacterianos que pueden permanecer en la superficie radicular. Esta limitación puede atribuirse a varios factores, como la compleja anatomía de los dientes (por ejemplo, lesiones de furcación), las limitaciones mecánicas en relación con el tamaño de los instrumentos o la invasión de patógenos periodontales en los tejidos blandos adyacentes o por la posible recolonización de las bolsas periodontales desde otros sitios enfermos o nichos intraorales (3). Procedimientos complementarios como antibióticos liberados a nivel local o sistémico, han sido investigados (4). Sin embargo, un uso excesivo de antibióticos, responsables de la producción de organismos resistentes al medicamento y el difícil mantenimiento del antibiótico en una concentración terapéutica en la bolsa periodontal, han llevado a la búsqueda de enfoques antimicrobianos alternativos para regímenes de prevención y de tratamiento periodontal. Uno de estos enfoques es la terapia fotodinámica (TFD).

La TFD es un procedimiento que utiliza energía de la luz para activar un agente fotosensibilizante (fotosensibilizador) en presencia de oxígeno. El fotosensibilizador sufre una transición a un estado de energía superior, lo que conlleva a la generación de especies reactivas del oxígeno altamente citotóxicas, principalmente al oxígeno singlete, extremadamente tóxico para células y bacterias (5). Este oxígeno singlete causa diferentes efectos tóxicos sobre los microorganismos: daño de los lípidos de membrana, destrucción de proteínas y canales iónicos, eliminación de enzimas metabólicas críticas, aglutinación de células e inhibición directa de factores de virulencia exógenos, como lipopolisacárido, colagenasa y proteasa.

La principal ventaja de la TFD como modalidad de tratamiento antimicrobiano es su mecanismo bacte- 
ricida no específico que hace muy poco probable el desarrollo de resistencia a la acción citotóxica de oxígeno singlete o de los radicales libres. La TFD es igualmente eficaz contra bacterias resistentes a los antibióticos como a las susceptibles a los mismos, además la repetida fotosensibilización no induce selección de cepas resistentes (6).

Por otra parte, en la búsqueda de terapias eficaces para lograr una superficie radicular biocompatible, con mayor grado de descontaminación y reducción de la inflamación de los tejidos periodontales adyacentes, aparecen los láseres como terapia alternativa o coadyuvante de los métodos tradicionales. La capacidad de la radiación láser de causar mortalidad bacteriana (7) ha servido para concebir la técnica de la esterilización láser como una herramienta quirúrgica dentro del campo odontológico.

Gracias a los últimos avances, el intervalo de longitudes de onda de emisión de la radiación láser se ha visto ampliado considerablemente, y numerosos estudios sugieren que los láseres pueden ser aplicados en diferentes tipos de tratamientos dentales. Actualmente, existen numerosos láseres comerciales de uso en Periodoncia: Nd: YAG (acrónimo del inglés neodymiumdoped yttrium aluminium garnet), $\mathrm{CO}_{2}$, Er: YAG (acrónimo del inglés erbium-doped yttrium aluminium garnet), diodo... Cada láser tiene una aplicación terapéutica de elección, no pudiendo abordar de forma totalmente efectiva todos los tratamientos. La radiación láser, en función de su longitud de onda de emisión no se absorbe de igual forma en los tejidos, lo que produce una amplia gama de efectos relacionados con su absorción o profundidad de penetrado en el tejido. Diferentes estudios con diferentes sistemas láser muestran cómo el láser de Nd: YAG contribuye a la eliminación del cálculo dental y a la reducción de los microbios endodónticos (8); también los láseres de diodo y de $\mathrm{CO}_{2}$ han mostrado alto poder desinfectante (9). Más recientemente se ha introducido el láser de Er: YAG por su versatilidad en la ablación de tejidos duros y blandos (10) y por su demostrada capacidad para eliminar no sólo bacterias sino también toxinas bacterianas difundidas por el cemento radicular (11). Por último, láseres de diodo de baja potencia $(\sim \mathrm{mW})$ ejercen un efecto positivo disminuyendo el sangrado, profundidad de la bolsa y reduciendo niveles de metaloproteinasa-8 (MMP-8) en bolsa periodontal (12).
El medio activo del láser de Er:YAG es también un cristal sintético formado por itrio y aluminio con impurezas de erbio y estructura de granate. Su radiación pulsada e infrarroja emitiendo a 2.940 nanómetros, se caracteriza por ser muy bien absorbida por el agua, por lo tanto es particularmente indicada para una precisa y localizada ablación de los tejidos biológicos con alto contenido acuoso. El coeficiente de absorción del agua para el láser Er:YAG es teóricamente 10.000 y 15.000-20.000 veces mayor que el coeficiente para el láser $\mathrm{CO}_{2}$ y Nd:YAG, respectivamente. Además, como componente de la apatita, los grupos $\mathrm{OH}$ muestran su máxima absorción alrededor de $2.800 \mathrm{~nm}$, hecho que explica su capacidad de ablación sobre el esmalte, dentina y hueso, es decir, este láser está indicado tanto para tejidos blandos como duros.

Conviene resaltar que pese a la gran variedad de estudios publicados desde la introducción de la radiación láser en la terapéutica periodontal, apenas existen estudios que correlacionen el aspecto inflamatorio con el antimicrobiano de la enfermedad. Por otra parte, los datos de ensayos clínicos controlados que han evaluado el efecto de la TFD siguen siendo limitados. Por lo tanto, el objetivo del presente estudio clínico, controlado, a corto plazo, fue comparar las consecuencias clínicas, antiinflamatorias y antimicrobianas del desbridamiento convencional solo o complementado con TFD o con la radiación láser de Er:YAG.

\section{MATERIAL Y MÉTODOS}

\section{Diseño del estudio y población estudiada}

Durante el periodo comprendido entre marzo de 2007 a enero de 2009 , se estudiaron 45 pacientes (15 hombres y 20 mujeres), de 45 a 58 años de edad, con una edad media de $51 \pm 6$ años, con periodontitis crónica moderada-avanzada, reclutados del Departamento de Estomatología III de la Universidad Complutense de Madrid, y consecutivamente enrolados en el estudio entre marzo de 2008 a enero de 2009. Como criterios de inclusión se consideraron: presencia de al menos 4 dientes por cuadrante, en los cuales no hubiera coronas, puentes o apoyo de prótesis removible, ni amalgamas tipo II o IV. Presencia de al menos 6 dientes con una profundidad de sondaje $>4 \mathrm{~mm}$ y una pérdida de hueso radiográfica 
(de 30\% en los lugares relacionados), de acuerdo a la clasificación de severidad periodontal basada en la localización de la cresta alveolar. Ausencia de lesiones de furca tipo III. Como criterios de exclusión se consideraron: pacientes fumadores o anteriormente fumadores, antecedentes de tratamiento periodontal previo, terapia con antibióticos o antiinflamatorios recibida en los últimos tres meses anteriores al tratamiento o durante el tratamiento, uso de colutorios antisépticos, corticosteroides o inmunosupresores, o cualquier condición sistémica que pudiera afectar al periodonto o el protocolo de tratamiento.

Los 45 participantes recibieron tratamiento periodontal básico incluyendo raspado y alisado radicular (RAR) e instrucciones de higiene oral. Además, 15 pacientes aleatoriamente asignados, recibieron tratamiento complementario con terapia fotodinámica (RAR+TFD). Del mismo modo, otros 15 recibieron tratamiento complementario con la radiación de un láser Er:YAG $(\lambda=2.940 \mathrm{~nm})(\mathrm{RAR}+\mathrm{ERL})$. Todos los pacientes fueron tratados por el mismo operador experimentado (A.D). Para asegurar el carácter ciego del estudio, los pacientes fueron tratados por un examinador (A.G) y un operador (A.D). Además las muestras tomadas se cegaron en el laboratorio donde se procesaron.

En cuanto a los requisitos éticos, una vez explicado el tratamiento por los operadores, los pacientes de forma voluntaria decidieron enrolarse en el estudio mediante la firma del Consentimiento Informado del estudio de investigación. Este estudio cuenta con la aprobación de la Unidad de Coordinación de Ensayos Clínicos del Hospital San Carlos de Madrid.

\section{Programa de higiene oral}

Antes de realizar el tratamiento en ambos grupos, los pacientes fueron instruidos en la técnica de cepillado de Bass así como en la capacidad de diferenciar la presencia o ausencia de placa bacteriana en las superficies dentales.

Además dependiendo de las exigencias de cada paciente, instruimos en la utilización de cepillos interproximales o seda dental. Los pacientes no utilizaron colutorios durante el estudio, para no modificar los resultados obtenidos.

\section{Mediciones clínicas: porcentaje de placa en boca total, porcentaje de sangrado en boca total y profundidad de sondaje}

Los parámetros clínicos fueron estudiados en todos los dientes, (excluyendo los terceros molares), en seis localizaciones: mesiovestibular, vestibular, distovestibular, mesiolingual, lingual y distolingual. Se calculó el Porcentaje de placa en boca total (PPBT), Porcentaje de sangrado en boca total (PSBT) y Profundidad de Sondaje (PS) antes de realizar el tratamiento correspondiente a cada grupo.

El PPBT fue determinado mediante una sonda periodontal en seis localizaciones de cada diente y se registró ausencia o presencia de la misma (Índice modificado de $\mathrm{O}$ ’Leary y cols).

La PS fue obtenida con la ayuda de una sonda periodontal calibrada (CP 15 UNC; Hu-Friedy Instrument Co, Chicago, IL, USA). El PSBT se obtuvo simultáneamente a la PS, basándonos en la presencia o ausencia de sangrado después de $30 \mathrm{~s}$ tras el sondaje.

Dichos parámetros clínicos se anotaron tanto antes de realizar los tratamientos correspondientes a cada grupo, así como 4 y 8 semanas después del tratamiento, de la misma forma descrita con anterioridad y en las mismas localizaciones en cada uno de los pacientes.

\section{Recolección de Fluido Crevicular Gingival (FCG)}

La localización seleccionada para la toma de fluido fue la bolsa más profunda de cada cuadrante, tomando así 4 muestras en 4 bolsas periodontales de cada paciente del estudio, excluyendo los terceros molares para evitar contaminación por saliva. La toma de muestras se efectuó al día siguiente de la profilaxis supragingival y del periodontograma para evitar el sangrado y la contaminación de las puntas en la toma de muestras.

Una vez aislado el diente con rollos de algodón se eliminó la placa supragingival con sonda periodontal sin tocar la encía marginal para una correcta extrac- 
ción de la muestra. La superficie dentaria fue secada con una jeringa de aire y se recolectó el FCG con puntas de papel prefabricadas (Periopaper, Oraflow INC., Plainview, NY, USA), las cuales fueron introducidas en las bolsas periodontales, hasta que la resistencia lo permitiera y se dejaron durante 30 segundos absorbiendo dicho líquido. Las muestras contaminadas de sangre fueron desechadas.

Después de la recogida de FCG, el volumen de la muestra fue medido con Periotron 8000 (Pro-Flow Inc., Amityville, New Cork, USA). Las lecturas del Periotron 8000 fueron convertidas en volumen actual $(\mu \mathrm{l})$, previa realización de una curva de calibrado realizada con suero humano de pacientes sanos (donantes de sangre) y ajustada a una regresión polinómica de cuarto orden (13).

Las puntas de periopaper fueron almacenadas en viales estériles con filtro y congeladas a $-80^{\circ} \mathrm{C}$ para su posterior análisis.

Las muestras se tomaron antes y a las 4 y 8 semanas del tratamiento para analizar los niveles de IL- $1 \beta$ y FNT- $\alpha$ en FCG y la variación de la capacidad antioxidante total del FCG.

\section{Muestras de placa subgingival}

Las muestras de placa subgingival fueron recolectadas en localizaciones muy cercanas a las de recogida del FCG.

Una vez aislada la localización con rollos de algodón y secada con jeringa de aire, los depósitos supragingivales fueron eliminados cuidadosamente con sonda periodontal. Las muestras de microbiota subgingival se obtuvieron introduciendo 2 puntas de papel estériles N 30 (Dentsply) hasta el nivel más profundo de la bolsa periodontal durante 30 segundos, evitando una posible contaminación con saliva.

Las puntas de papel de cada localización fueron introducidas en viales estériles conteniendo 1,5 ml de medio de transporte RTF y se enviaron al Laboratorio de Microbiología de la Facultad de Odontología de la Universidad Complutense de Madrid, para el análisis mediante cultivo.

\section{Técnicas de tratamiento}

\section{Tratamiento periodontal básico}

Los 45 sujetos enrolados en la investigación (quince pacientes del grupo RAR, quince pacientes del grupo RAR+TFD y quince del grupo RAR+ERL) recibieron un tratamiento periodontal básico de la enfermedad periodontal crónica moderada/avanzada, que consistió en la eliminación del cálculo supragingival y subgingival.

La eliminación del cálculo supragingival se realizó con ultrasonidos sonicador SONYflex 2003 de Kavo y punta accesoria universal, y posteriormente se aplicó una pasta abrasiva (Dentsply) mediante un cepillo profilaxis de cerda natural (Stoddard, diámetro $8 \mathrm{~mm}$ para contraángulo).

La eliminación del cálculo subgingival se realizó de forma manual mediante curetas (Hu-Friedy Co., Chicago, IL, USA) bajo anestesia con lidocaína 2\% (Octocaine 1:100.000, Laboratorios Clarben S.A, Madrid), durante al menos $30 \mathrm{~min} /$ cuadrante o hasta que el operador considerara la correcta eliminación del cálculo.

\section{Terapia fotodinámica}

El fotosensibilizador utilizado consistió en 3,7-bis (dimetil-amino) cloruro de fenazationio (azul de metileno) $0,005 \%$ (p/v), suspendido en una solución de tampón de fosfato salino con hidroximetilcelulosa como agente de viscosidad mucoadhesivo (Tratamiento PeriowaveTM Kit, Ondine BIOPHARMA Corporation, Vancouver, BC, Canadá). La solución fotosensibilizante se aplicó directamente en toda la boca con un Dentsply (York, PA, EE.UU.) de calibre 23, acabado en una aguja de irrigación para permitir la irrigación completa de la parte coronal de la bolsa periodontal. La fotoactivación se llevó a cabo utilizando un láser de diodo (PeriowaveTM, Ondine BioPharma) $(\lambda=670 \mathrm{~nm})$ con una potencia máxima de $200 \mathrm{~mW}$. La radiación se aplica a través de una fibra óptica flexible conectada a una pieza de mano autoclavable de acero inoxidable diseñada para el usuario. La pieza de mano da cabida a una punta difusora de luz configurada de manera similar a una sonda periodontal para permitir el acceso a la bolsa 
periodontal. Todos los tratamientos se realizaron con una potencia continua de $200 \mathrm{~mW}$ (comprobada antes de cada tratamiento mediante el empleo de un detector de potencia láser XLP12 de Gentec en combinación con una consola Gentec-SOLO 2) y tiempo de irradiación de 60 segundos por sitio. La punta difusora de luz se movió suavemente alrededor de la bolsa durante el ciclo de la iluminación. Paciente, operador, y los asistentes utilizaron gafas de protección durante la aplicación láser.

Todos los grupos fueron tratados con anestesia local (lidocaína 2\%, octocaína 1:100.000, Laboratorios Clarben SA, Madrid).

\section{Tratamiento con láser de Er: YAG}

Los quince pacientes ubicados en el grupo RAR+ERL, recibieron la aplicación complementaria de la radiación infrarroja de un láser de Er:YAG (KEY 3+; KaVo, Biberach, Alemania) emitiendo a una longitud de onda de $2.940 \mathrm{~nm}$, combinado con un sistema de detección de cálculo dental (mediante la señal de fluorescencia inducida en los tejidos mineralizados irradiados con un láser diodo de InGaAsP emitiendo a $655 \mathrm{~nm}$ ). La energía del pulso utilizada fue de $160 \mathrm{~mJ}$ (medida con un detector piroeléctrico Gentec-DE $500+$ en combinación con una consola GentecSOLO) y una velocidad de repetición de $10 \mathrm{~Hz}$, con irrigación de agua destilada de acuerdo a las instrucciones del fabricante. La introducción del láser en la bolsa periodontal se realizó mediante una pieza especialmente diseñada (2261, KaVo, Biberach, Alemania) y una fibra de vidrio biselada (tamaño $0,4 \mathrm{~mm} \mathrm{x}$ 1,65 mm, factor de transmisión: 0.81) ("blue light wedge”, KaVo, Biberach, Alemania). El tratamiento se efectuó en sentido coronoapical con una inclinación de la fibra de vidrio de $15^{\circ}-20^{\circ}$ respecto a la superficie radicular (14). El tiempo de exposición utilizado por cada superficie de diente (vestibular y lingual) fue de $30 \mathrm{~s}$ (en total $1 \mathrm{~min}$. por diente).

\section{Procesado de las muestras}

Las muestras fueron analizadas en el Laboratorio de Investigación de la Facultad de Odontología, Universidad Complutense, Madrid.

\section{Medición de IL-1ß y TNF- $\alpha$}

Las muestras diluidas de FGC fueron analizadas para determinar la concentración de mediadores proinflamatorios mediante la técnica ELISA (ensayo inmunoabsorbente ligado a enzimas). Los niveles de IL- $1 \beta$ y TNF- $\alpha$ en FCG fueron determinados por duplicado mediante BLK-223 (kit de ELISA para determinación del TNF- $\alpha$ humano) y BLK-224 (kit de ELISA para la determinación de IL-1 $\beta$ ) respectivamente, ambos de Biolink (Biolink 2000, Barcelona, España), siguiendo las instrucciones del fabricante. Los resultados se calcularon a partir de las curvas estándar creadas para cada estudio. La concentración de citoquina en una determinada posición se calculó dividiendo la cantidad de citoquina determinada por ELISA entre el volumen de FCG extraído correspondiente a esa misma posición, previamente calculado a partir de la curva de calibrado del Periotron 8000, y por lo tanto se expresó en $\mathrm{pg} / \mu \mathrm{l}$.

\section{Estado antioxidante total}

La determinación de la capacidad antioxidante total se midió mediante el test fotométrico (ImAnOx (TAS) Kit, Immundiagnostik AG, Stubenwald-Allee 8a, D 64625 Bensheim). Esta determinación se llevó a cabo mediante la reacción de los antioxidantes de la muestra con una cantidad definida de peróxido de hidrógeno proporcionado exógenamente $\left(\mathrm{H}_{2} \mathrm{O}_{2}\right)$. Los antioxidantes de la muestra eliminan una cantidad determinada del $\mathrm{H}_{2} \mathrm{O}_{2}$ proporcionado, de manera que el $\mathrm{H}_{2} \mathrm{O}_{2}$ residual se determina fotométricamente mediante la reacción enzimática que implica la conversión de (3,3',5-5' -tetrametilbencidina (TMB) en un producto coloreado. Las muestras se miden a 450 $\mathrm{nm}$, y son expresadas en $\mu \mathrm{mol} / \mathrm{l}$.

\section{Cultivo microbiológico}

Las muestras de placa subgingival fueron dispersadas mediante un vortex (Thermolyne Maxi Mix II Tipo de 37.600, IA, EE.UU.) durante 45 s. Se realizaron una serie de diluciones décimo normales $\left(10^{-0}, 10^{-1}, 10^{-2}\right.$, $10^{-3}, 10^{-4}$ y $10^{-5}$ ) en medio PBS (tampón fosfato salino) de la suspensión bacteriana. Alícuotas de $100 \mu \mathrm{l}$ de una adecuada dilución $\left(10^{-3}, 10^{-4}\right.$ y $\left.10^{-5}\right)$ se deposi- 
taron en medios de cultivo de agar-sangre (Oxoid $n^{\circ}$ 2, Oxoid Ltd, Basingstoke, Reino Unido). Las placas se incubaron a $37^{\circ} \mathrm{C}$ durante $7-14$ días en atmósfera anaeróbica para la detección y cuantificación de Porphyromonas gingivalis $(\mathrm{Pg})$, Prevotella intermedia (Pi), Parvimonas Micra (Pm), Fusobacterium nucleatum (Fn), Campylobacter rectus (Cr) Eikenella corrodens (Ec), Tannerella forsythia (Tf), Capnocytophaga species y Eubacterium nodatum (En).

Las bacterias fueron identificadas principalmente atendiendo a la morfología de las colonias utilizando un microscopio estereoscópico Kyowa SDZ-PL (Kyowa Optical, Tokio, Japón), a la producción de pigmentos y a la tinción de Gram.

Una cantidad de $100 \mathrm{ml}$ de muestras sin diluir y de diluidas $10^{-1}$ fueron inoculadas en las placas de agar selectivo para Aggregatibacter actinomycetemcomitans (Aa) (Dentaid-1 media) y se incubaron a $37^{\circ} \mathrm{C}$ durante 2-3 días en una atmósfera con un 5\% de $\mathrm{CO}_{2}$. La tinción Gram y la producción de catalasa también se utilizaron para la identificación de Aa.

El recuento total de colonias y el número total de bacterias (unidades formadoras de colonias por mililitro de líquido extraído (UFC/mL)) fueron evaluados 14 días después de la inoculación.

\section{Análisis estadístico}

Se procedió al análisis estadístico descriptivo de los resultados obtenidos (media y desviación típica). Se analizaron las diferencias en la evolución de los parámetros clínicos, microbiológicos e inmunológicos (antes del tratamiento, a las 4 y 8 semanas) entre los tres diferentes tipos de tratamientos aplicados: RAR, RAR+TFD y RAR+ERL.

Para establecer comparaciones intragrupos (entre post-terapia y estado basal), el test utilizado para el análisis estadístico fue el test no paramétrico de Wilcoxon, considerándose los valores de $\mathrm{p}<0.05$ como estadísticamente significativos. Para establecer comparaciones intergrupos, el test utilizado fue el análisis de las varianzas (ANOVA) y para determinar las diferencias entre grupos se procedió a realizar pruebas a posteriori mediante el test Duncan.
Los valores $\mathrm{p}<0.05$ fueron aceptados como estadísticamente significativos.

\section{RESULTADOS}

\section{Estudio de índices clínicos}

En primer lugar, se procedió a evaluar la evolución del PPBT (Figura 1A) durante el periodo estudiado. A las 4 semanas del tratamiento se observó una marcada reducción de dicho índice disminuyendo de manera más suave en el periodo comprendido entre 4 semanas hasta las 8 semanas finales del tratamiento en los grupos tratados con RAR y RAR+TFD. El grupo tratado con RAR+ERL no experimentó reducción entre las 4 y 8 semanas. No se encontraron diferencias significativas entre los tres grupos estudiados.

En cuanto a la evolución del PSBT (Figura 1B) se observó cómo su reducción también fue marcada a las 4 semanas del tratamiento en los tres grupos de estudio. Se observó en el grupo tratado con RAR+TFD un considerable descenso de PSBT a las 8 semanas, aunque las diferencias intergrupos no fueron significativas.

Por otra parte, de los datos de profundidad de sondaje (PS) representados en la Figura 1C, se deduce que los tres tipos de terapia consiguieron reducir la PS durante el tratamiento. A las 4 semanas, la reducción de PS fue estadísticamente significativa en el grupo tratado con RAR+ERL. Sin embargo, a las 8 semanas del tratamiento se observó una reducción más acusada en los grupo tratados con RAR y RAR+TFD frente a la obtenida con el tratamiento RAR+ERL, aunque no significativa estadísticamente.

\section{Estudio microbiológico}

El análisis de la flora total subgingival para cada tratamiento en cada período de seguimiento no indica diferencias significativas entre los grupos de tratamiento (Tabla 1), aunque se encontraron diferencias estadísticamente significativas $(p<0,05)$ intragrupos antes del tratamiento y entre 4 y 8 semanas (Tabla 1 ).

El número de sitios colonizados por especie en cada período del estudio para los tres tratamientos reali- 


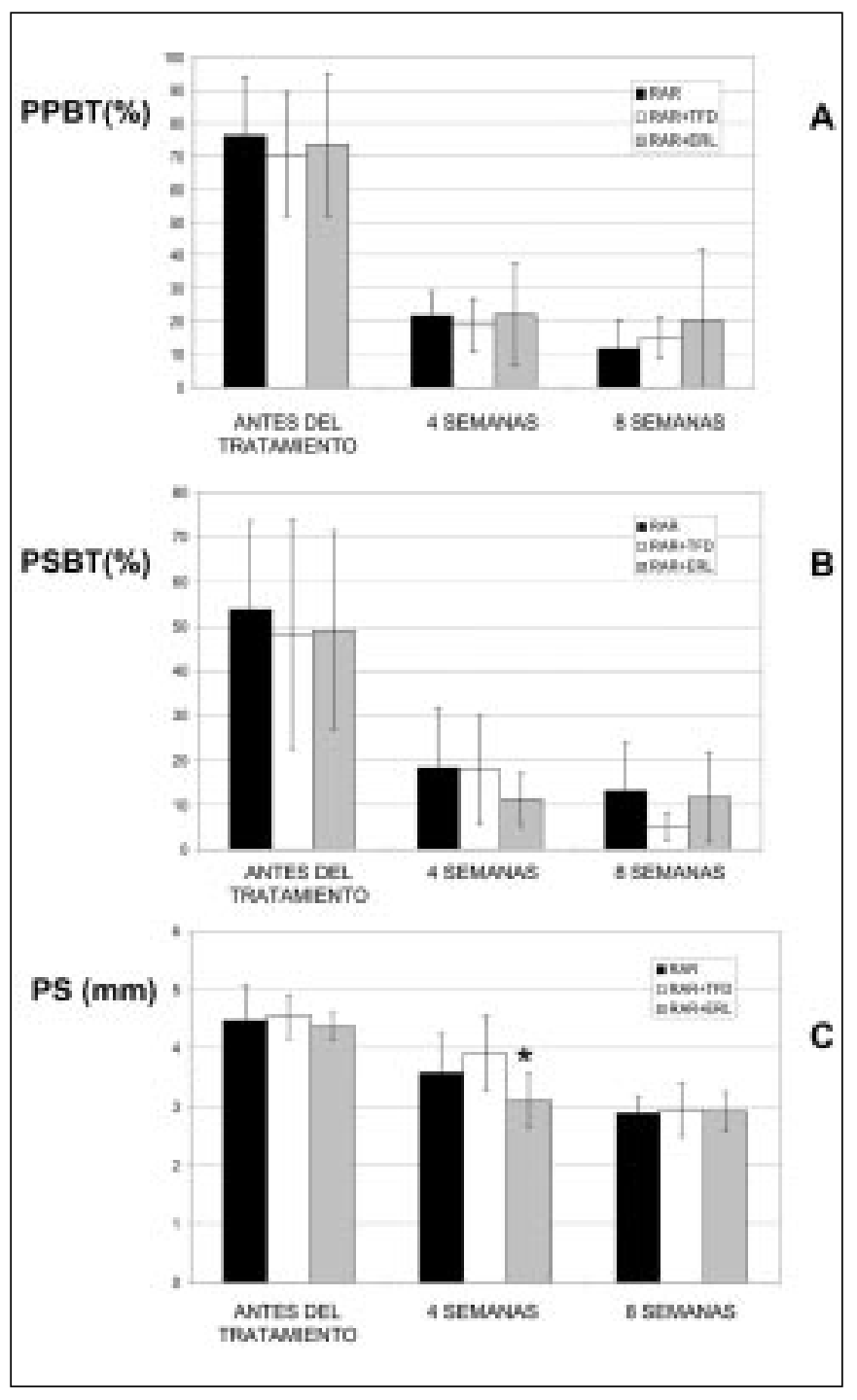

Fig. 1. A) Porcentaje de placa en boca total (PPBT), B) Porcentaje de sangrado en boca total (PSBT) y C) Profundidad de sondaje (PS): Media \pm Desv. tip., ( $n=45$ pacientes) antes del tratamiento y a las 4 y 8 semanas post-terapia. (*) Diferencias estadísticamente significativas entre los grupos en cuanto a la evolución de los parámetros clínicos estudiados analizadas mediante el test de Duncan $(p<0,05)$.

zados se recoge en la Tabla 2. En general, cada una de las especies evaluadas se redujo 4 semanas después de la terapia, y se observó un pequeño aumento tras 8 semanas. En los grupos RAR+ERL y RAR+TFD, Fn fue la especie más prevalente durante todo el estudio, mientras que en el grupo de RAR, $P i$ fue la especie más prevalente antes del tratamiento y de nuevo Fn fue la especie más frecuente durante el estudio.

Analizando la Tabla 3, observamos que en los tres grupos la mayoría de las localizaciones estaban infectadas por 3 y 4 especies antes del tratamiento, en el grupo RAR $75 \%$ de las localizaciones, en grupo RAR+ERL $61 \%$ y en el grupo RAR+TFD 55\%. A las 4 semanas la mayoría de las localizaciones (36\%) estaban colonizadas por una sola especie en el grupo RAR, mientras que un $25 \%$ en el grupo RAR+ERL y un $16 \%$ en el grupo RAR+TFD, aparecían colonizadas también por 1 especie. A las 8 semanas del tratamiento tan solo un $8 \%$ de las localizaciones seguían estando colonizadas por una especie en el grupo RAR, un $13 \%$ en el grupo RAR+TFD y un $28 \%$ en el grupo RAR+ERL. Por lo tanto, no se observó ventaja adicional después del tratamiento de fotodesinfección llevado a cabo mediante TFD en relación a la recolonización bacteriana frente a RAR, sin embargo en el grupo

\section{TABLA 1.- FLORA SUBGINGIVAL TOTAL: MEDIA \pm DESV. TIP., (N=45 PACIENTES) ANTES DEL TRATAMIENTO, A LAS 4 Y 8 SEMANAS POST-TERAPIA}

\begin{tabular}{|l|c|c|c|c|c|c|c|c|}
\hline & \multicolumn{2}{|c|}{$\begin{array}{c}\text { Antes del } \\
\text { tratamiento }\end{array}$} & \multicolumn{3}{|c|}{4 semanas } & \multicolumn{2}{c|}{8 semanas } \\
\hline & Media & $D E$ & Media \pm DE & Diferencia & Valor $P^{*}$ & Media $\pm D E$ & Diferencia & Valor $P^{*}$ \\
\hline RAR & $8,5 \cdot 10^{5}$ & $8,1 \cdot 10^{5}$ & $9,1 \cdot 10^{4} \pm 6,2 \cdot 10^{4}$ & $7,6 \cdot 10^{5}$ & $<0,05$ & $8 \cdot 10^{4} \pm 3,9 \cdot 10^{4}$ & $7.7 \cdot 10^{5}$ & $<0,05$ \\
RAR+TFD & $2,3 \cdot 10^{5}$ & $1,9 \cdot 10^{5}$ & $3,3 \cdot 10^{4} \pm 1,1 \cdot 10^{4}$ & $2 \cdot 10^{5}$ & $<0,05$ & $3,5 \cdot 10^{4} \pm 6,7 \cdot 10^{4}$ & $2 \cdot 10^{5}$ & $<0,05$ \\
RAR+ERL & $1,5 \cdot 10^{6}$ & $1,4 \cdot 10^{6}$ & $1,1 \cdot 10^{5} \pm 9,9 \cdot 10^{4}$ & $1,4 \cdot 10^{6}$ & $<0,05$ & $6,8 \cdot 10^{4} \pm 6,5 \cdot 10^{4}$ & $1,4 \cdot 10^{6}$ & $<0,05$ \\
Valor P** & & & & NS & & & NS & \\
\hline
\end{tabular}

* Diferencias estadísticamente significativas dentro de cada grupo (comparando con el estado inicial antes del tratamiento) a diferentes tiempos mediante el test no-paramétrico de Wilcoxon: $(p<0,05)$.

** Diferencias estadisticamente significativas entre los grupos a diferentes tiempos mediante el test de Duncan: $(p<0,05)$.

$N S=$ no significativa. 


\begin{tabular}{|c|c|c|c|c|}
\hline & \multicolumn{4}{|c|}{$\begin{array}{c}\text { TABLA 2.- NÚMERO DE SITIOS } \\
\text { COLONIZADOS POR LAS ESPECIES } \\
\text { EVALUADAS ANTES Y DESPUÉS DE } \\
\text { CADA TRATAMIENTO } \\
\text { (No TOTAL DE SITIOS ESTUDIADOS } \\
\text { POR GRUPO: 60). } \\
\text { EN "NEGRITA" LOS VALORES MÁS } \\
\text { ALTOS ALCANZADOS }\end{array}$} \\
\hline & & $\begin{array}{c}\text { Antes del } \\
\text { tratamiento }\end{array}$ & $\begin{array}{c}4 \\
\text { sem. }\end{array}$ & $\begin{array}{c}8 \\
\text { sem }\end{array}$ \\
\hline Aa & $\begin{array}{l}\text { RAR } \\
\text { RAR+TFD } \\
\text { RAR+ERL }\end{array}$ & $\begin{array}{r}0 \\
0 \\
10\end{array}$ & $\begin{array}{l}0 \\
0 \\
0\end{array}$ & $\begin{array}{l}0 \\
0 \\
5\end{array}$ \\
\hline$P g$ & $\begin{array}{l}\text { RAR } \\
\text { RAR+TFD } \\
\text { RAR+ERL }\end{array}$ & $\begin{array}{l}40 \\
25 \\
40\end{array}$ & $\begin{array}{l}20 \\
18 \\
23\end{array}$ & $\begin{array}{l}15 \\
23 \\
17\end{array}$ \\
\hline$P i$ & $\begin{array}{l}\text { RAR } \\
\text { RAR+TFD } \\
\text { RAR+ERL }\end{array}$ & $\begin{array}{l}55 \\
45 \\
48\end{array}$ & $\begin{array}{l}20 \\
42 \\
35\end{array}$ & $\begin{array}{l}15 \\
45 \\
27\end{array}$ \\
\hline$P m$ & $\begin{array}{l}\text { RAR } \\
\text { RAR+TFD } \\
\text { RAR+ERL }\end{array}$ & $\begin{array}{l}15 \\
28 \\
18\end{array}$ & $\begin{array}{r}0 \\
13 \\
5\end{array}$ & $\begin{array}{r}15 \\
18 \\
7\end{array}$ \\
\hline$F n$ & $\begin{array}{l}\text { RAR } \\
\text { RAR+TFD } \\
\text { RAR+ERL }\end{array}$ & $\begin{array}{l}55 \\
53 \\
50\end{array}$ & $\begin{array}{l}45 \\
43 \\
60\end{array}$ & $\begin{array}{l}55 \\
50 \\
55\end{array}$ \\
\hline $\mathrm{Cr}$ & $\begin{array}{l}\text { RAR } \\
\text { RAR+TFD } \\
\text { RAR+ERL }\end{array}$ & $\begin{array}{r}0 \\
10 \\
5\end{array}$ & $\begin{array}{l}0 \\
3 \\
0\end{array}$ & $\begin{array}{l}0 \\
5 \\
3\end{array}$ \\
\hline$E c$ & $\begin{array}{l}\text { RAR } \\
\text { RAR+TFD } \\
\text { RAR+ERL }\end{array}$ & $\begin{array}{l}20 \\
20 \\
15\end{array}$ & $\begin{array}{r}10 \\
15 \\
8\end{array}$ & $\begin{array}{l}15 \\
15 \\
10\end{array}$ \\
\hline$T f$ & $\begin{array}{l}\text { RAR } \\
\text { RAR+TFD } \\
\text { RAR+ERL }\end{array}$ & $\begin{array}{l}25 \\
13 \\
10\end{array}$ & $\begin{array}{l}0 \\
3 \\
0\end{array}$ & $\begin{array}{l}5 \\
3 \\
3\end{array}$ \\
\hline $\begin{array}{l}\text { Cap. } \\
\text { spp. }\end{array}$ & $\begin{array}{l}\text { RAR } \\
\text { RAR+TFD } \\
\text { RAR+ERL }\end{array}$ & $\begin{array}{l}20 \\
10 \\
12\end{array}$ & $\begin{array}{l}0 \\
3 \\
3\end{array}$ & $\begin{array}{r}15 \\
8 \\
5\end{array}$ \\
\hline En & $\begin{array}{l}\text { RAR } \\
\text { RAR+TFD } \\
\text { RAR+ERL }\end{array}$ & $\begin{array}{l}0 \\
0 \\
3\end{array}$ & $\begin{array}{l}0 \\
0 \\
3\end{array}$ & $\begin{array}{l}0 \\
0 \\
3\end{array}$ \\
\hline
\end{tabular}

RAR+ERL se puede observar un retraso en la recolonización bacteriana respecto al resto de grupos.

\section{Estudio antiinflamatorio}

Se procedió a la evaluación de la concentración de IL- $1 \beta$ y TNF- $\alpha$ en bolsa periodontal antes del tratamiento y a las 4 y 8 semanas después, cuyos resultados se muestran en las Figuras $2 a$ y $2 b$ respectivamente. En cuanto al estudio de la IL-1 $\beta$, el grupo RAR mostró un incremento de la concentración a las 4 semanas y un mantenimiento de dicha concentración a las 8 semanas (Figura 2A). También, dentro de este mismo grupo, observamos cómo los niveles de TNF- $\alpha$ en FCG aumentan suavemente a las 4 semanas respecto al estado inicial de los pacientes, y cómo el incremento se hace más acusado durante las siguientes semanas, llegando a alcanzar valores bastante más altos que los iniciales dos meses después del tratamiento (Figura 2B).

Por otra parte, el grupo tratado con RAR+ERL mostró una importante reducción de la concentración de IL-1 $\beta$ a las 4 semanas del tratamiento, estableciéndose diferencias estadísticamente significativas entre este grupo y los grupos RAR y RAR+TFD en cuanto a la concentración de IL- $1 \beta$ entre las 0 y 4 semanas y 0 y 8 semanas $(p<0,00)$. El grupo tratado con RAR+TFD, mostró un ligero incremento en el nivel de esta citoquina en FCG a las 4 semanas post-tratamiento. Entre las 4 y 8 semanas siguientes al tratamiento, sólo en el grupo RAR+ERL la concentración de IL- $1 \beta$ se ve muy ligeramente incrementada (Figura 2A), frente a su mantenimiento en el grupo RAR+TFD.

En cuanto a la evolución de la concentración de TNF$\alpha$, en el grupo RAR+ERL podemos observar cómo disminuye ligeramente a las 4 semanas y se mantiene constante durante las 4 semanas finales. En el grupo RAR+TFD los niveles de TNF- $\alpha$ se incrementan muy lentamente durante todo el post-tratamiento. El análisis estadístico muestra diferencias estadísticamente significativas en cuanto a la evolución de los niveles de TNF- $\alpha$ en bolsa periodontal en grupo RAR+ERL frente a los grupos RAR y RAR+TFD a las 4 semanas posterapia $(p<0,00)$ y del grupo RAR frente a los grupos complementados (RAR+ERL y $R A R+T F D)$ a las 8 semanas posterapia $(p<0,00)$. 


\begin{tabular}{|c|c|c|c|c|c|c|c|c|c|c|c|c|c|c|c|}
\hline \multicolumn{16}{|c|}{$\begin{array}{c}\text { TABLA 3.- PORCENTAJE DE SITIOS INFECTADOS POR 1, 2, 3, } 4 \text { O } 5 \text { ESPECIES ANTES DE } \\
\text { CADA TRATAMIENTO Y A LAS } 4 \text { Y } 8 \text { SEMANAS POST-TERAPIA. EN "NEGRITA" LOS } \\
\text { VALORES MÁS ALTOS. }\end{array}$} \\
\hline & \multicolumn{3}{|c|}{1 especie } & \multicolumn{3}{|c|}{2 especies } & \multicolumn{3}{|c|}{3 especies } & \multicolumn{3}{|c|}{4 especies } & \multicolumn{3}{|c|}{5 especies } \\
\hline & RAR & $\mathrm{R}+\mathrm{TF}$ & R+ERL & RAR & $\mathrm{R}+\mathrm{TFD}$ & $\mathrm{R}+\mathrm{ERL}$ & RAR & $\mathrm{R}+\mathrm{TF}$ & $\mathrm{R}+\mathrm{ERL}$ & RAR & $\mathrm{R}+\mathrm{TF}$ & $\mathrm{R}+\mathrm{ERL}$ & RAR & $\mathrm{R}+\mathrm{TFD}$ & $\mathrm{R}+\mathrm{ERL}$ \\
\hline $\begin{array}{l}\text { Antes del } \\
\text { tratamiento }\end{array}$ & 0 & 16 & 3 & 8 & 16 & 22 & 33 & 22 & 22 & 42 & 33 & 39 & 17 & 13 & 14 \\
\hline 4 semanas & 36 & 16 & 25 & 0 & 33 & 33 & 17 & 22 & 17 & 17 & 21 & 14 & 0 & 8 & 11 \\
\hline 8 semanas & 8 & 13 & 28 & 42 & 33 & 19 & 25 & 21 & 22 & 8 & 21 & 17 & 17 & 12 & 14 \\
\hline
\end{tabular}

La figura 3 muestra la evolución de la capacidad antioxidante durante el post-tratamiento. No se han encontrado diferencias estadísticamente significativas entre los tres tratamientos a lo largo de todo el estudio, ya que la capacidad antioxidante del FCG se mantiene casi constante durante todo el estudio, apenas aumentado de manera muy sutil a las 4 semanas en los tres grupos evaluados.

\section{DISCUSIÓN}

Los resultados del presente estudio demuestran que el tratamiento periodontal no quirúrgico solo o en combinación con la radiación láser de Er:YAG controlada por fluorescencia o complementado por TFD, fue bien tolerado y no se observaron complicaciones durante el periodo de estudio como abscesos o infecciones.

Las tres modalidades de tratamiento realizadas dieron lugar a importantes mejoras tras el tratamiento periodontal. Esta mejora fue más evidente en el caso de los parámetros clínicos, de manera que el tratamiento complementario tanto con la radiación láser de Er:YAG como con TFD, parece no conducir a un incremento adicional de la mejoría clínica del paciente en cuanto a los valores analizados en este estudio. Estos resultados concuerdan con los mostrados en un estudio clínico reciente realizado por Tomasi y cols. (15), donde se evaluaron los efectos de la terapia no quirúrgica en pacientes dentro de programas de mantenimiento, tratados bien con láser de Er: YAG o ultrasonidos, no mostró mejoría

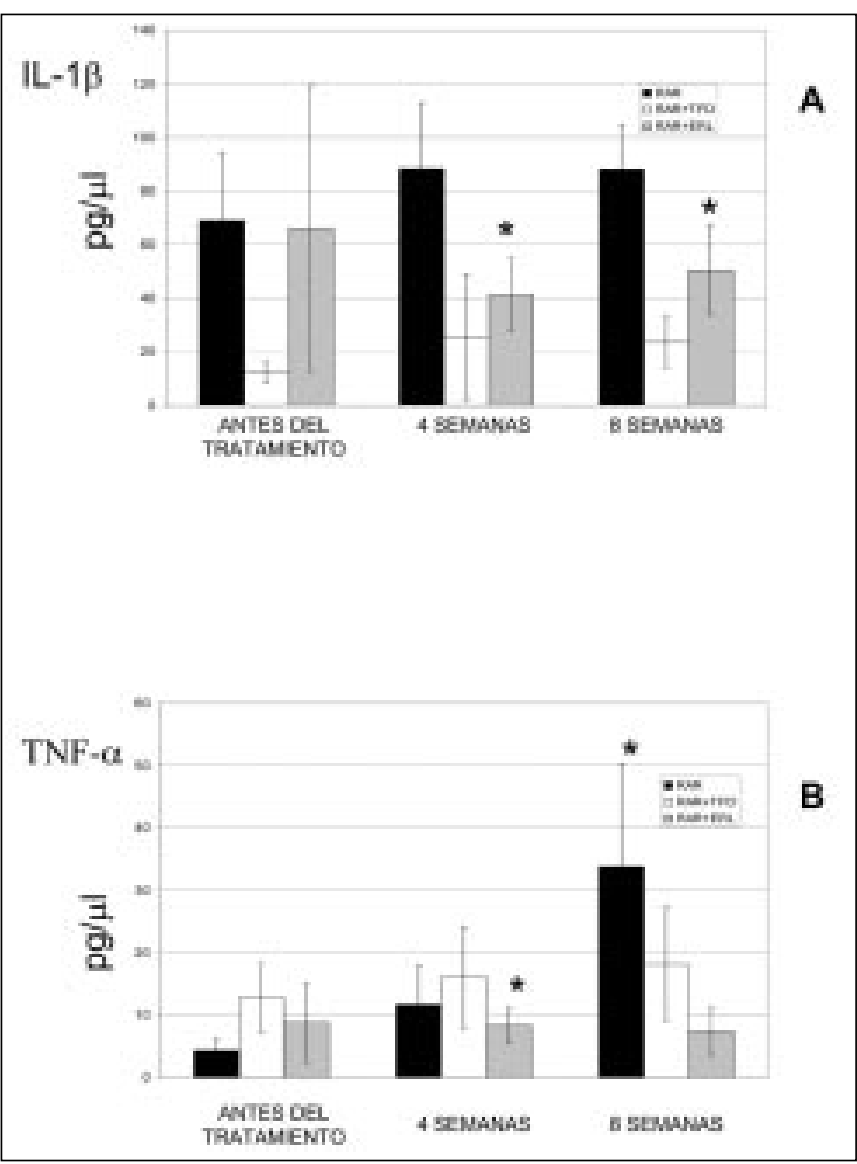

Fig. 2. A) Interleuquina $1 \beta$ (IL- $1 \beta)$ y B) Factor de necrosis tumoral- $\alpha$ (FNT- $\alpha$ ): Media \pm Desv. tip., $(n=45$ pacientes) antes del tratamiento y a las 4 y 8 semanas post-terapia. (*) Diferencias estadísticamente significativas entre los grupos en cuanto a la evolución de los niveles de citoquinas estudiadas en FCG analizadas mediante el test de Duncan $(p<0,05)$.

adicional con el tratamiento láser en cuanto a los parámetros clínicos evaluados. Jepsen y cols. (16), en un ensayo clínico controlado, trataron a 10 pacientes 


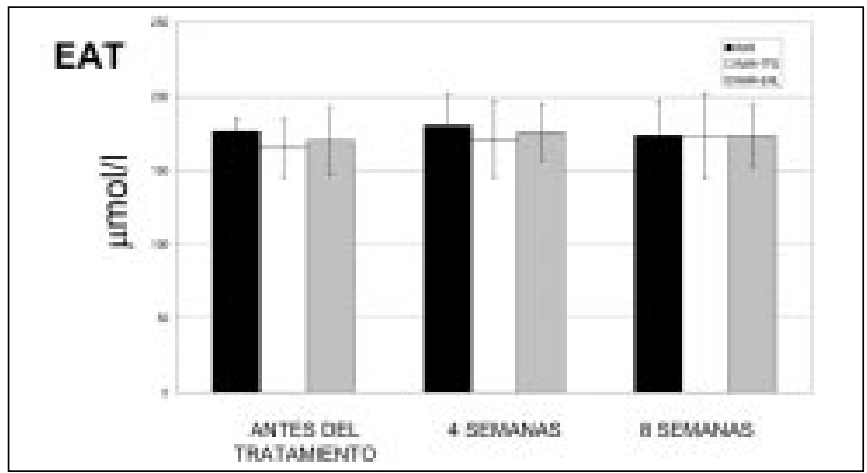

Fig. 3. Evolución del EAT del FGC: Media \pm Desv. tip., $(n=45$ pacientes) antes del tratamiento y a las 4 y 8 semanas post-terapia.

(*) Diferencias estadísticamente significativas entre los grupos en cuanto a la evolución de los niveles de citoquinas estudiadas en FCG analizadas mediante el test de Duncan $(p<0,05)$.

bien con RAR+ERL o bien con RAR, observando, al igual que en el presente estudio, diferencias estadísticamente significativas intragrupo, y no estadísticamente significativas entre los dos grupos en ninguno de los parámetros clínicos investigados. Considerando por otra parte los datos publicados en la literatura sobre aplicación complementaria de TFD al RAR, se observa una mayor reducción del sangrado al sondaje comparada con la obtenida tras la aplicación de RAR exclusivamente y similares reducciones en PS y en la pérdida de inserción $(17,19)$. Nosotros sí hemos detectado una considerable reducción en el PSBT, pero no significativa estadísticamente.

Los parámetros microbiológicos evaluados muestran que el uso de cualquiera de los tratamientos es eficaz, pero no causa la completa eliminación de bacterias. Las bacterias pueden residir en las irregularidades de la superficie radicular y en los túbulos dentinarios, así como en otros sitios de la cavidad oral, en particular en zonas extradentales, tales como la superficie dorsal de la lengua, mucosa bucal, paladar y amígdalas (20). Estas consideraciones deben tenerse en cuenta a la hora de interpretar los resultados antimicrobianos.

Los resultados obtenidos en el presente estudio muestran que la reducción microbiana tras los tratamientos con RAR+ERL y RAR+TFD es similar a la obtenida tras el RAR. El cambio más acentuado en la distribución de bacterias se produjo en el primer periodo de tiempo (es decir, a las 4 semanas post-tratamiento) con las tres modalidades realizadas. Estudios previos concuerdan con nuestros resultados tanto en la caso de la aplicación de RAR+ERL $(21,22)$, como tras la aplicación de RAR+TFD $(17,19)$. El número de especies patógenas albergadas en cada localización estudiada varió de 4 especies antes del tratamiento a 1 después de 4 semanas, y a 2-3 especies después de 8 semanas posterapia. Además, los resultados demuestran que la recolonización con 1 especie se mantiene durante más tiempo en el grupo RAR+ERL que en el grupo RAR+TFD, y en este último durante más tiempo que en el grupo RAR. El grupo tratado complementariamente con la radiación láser de Er:YAG controlada por fluorescencia, al contar con este sistema añadido para la detección selectiva del cálculo subgingival, permite prácticamente la entera eliminación del cálculo sin producir efectos mecánicos adicionales como una eliminación excesiva de cemento y una extremada irregularidad en la superficie radicular y por ello, dificultando el asentamiento bacteriano.

Se han observado asociaciones especificas microbianas entre complejos bacterianos diferentes: por ejemplo, es muy poco común encontrar especies del complejo rojo $(P g, T d, T f)$ en ausencia de miembros del complejo naranja (p.e. Pi) (23). El nuevo crecimiento y recolonización de las bolsas ocurre 3-7 días posttratamiento, pero con una clara alteración en la microflora subgingival (24). Los colonizadores primarios de la placa dental con frecuencia ocupan las bolsas periodontales más rápido, inhibiendo así el establecimiento de otras especies patógenas (25). Esto puede explicar por qué en este estudio hay más localizaciones infectadas por 1 ó 2 especies después de la terapia, mientras que la mayoría de las localizaciones albergan 3 ó 4 especies antes del tratamiento. Por otra parte, $F n$ fue el periodontopatógeno más frecuente entre las especies evaluadas tras la realización de los tres procedimientos estudiados, actuando como bacteria puente en el proceso de recolonización, favoreciendo así la formación de biofilm gracias a la coagregación de todas las bacterias bucales (26).

Aunque la mayoría de los estudios clínicos publicados muestran la disminución de la concentración de citoquinas en surco periodontal después del tratamiento periodontal no quirúrgico (RAR), especialmente en cuanto a la reducción de la concentración de IL-1 $\beta$ $(27,29)$, nuestros resultados se aproximan más a los obtenidos por Yoshinari y cols. (30), en un estudio 
donde se investigó la relación entre los cambios clínicos e IL- $1 \beta$ después de la terapia periodontal convencional con RAR en pacientes con periodontitis crónica, observándose clara mejoría de los índices clínicos y ligero aumento de la concentración de IL-1ß.

A partir de estos resultados podemos constatar el poder antiinflamatorio de la fototerapia con la radiación de Er:YAG controlada por fluorescencia, que conduce a una inhibición en la producción de citoquinas, resultados que muestran concordancia con investigaciones previas, como la de Lopes y cols. (31), que valoraron el cambio en los niveles de Il- $1 \beta$ en FCG tras la aplicación de RAR+ERL y observaron, al igual que en nuestro estudio, cómo esta citoquina disminuía a los 12 días y a los 30 días post-tratamiento, aunque no de manera estadísticamente significativa respeto al procedimiento con RAR. Nuestros resultados demuestran cómo el tratamiento de RAR complementado con la radiación láser de Er:YAG promueve un descenso de la inflamación, con un descenso de los niveles de los mediadores inflamatorios estudiados en FCG, principalmente a las 4 semanas posttratamiento. La capacidad de este nuevo prototipo láser con radiación controlada por fluorescencia para detectar y eliminar con precisión el cálculo subgingival, especialmente cuando el cálculo se encuentra en la parte más profunda de la bolsa periodontal o en una superficie radicular con contornos anatómicos complejos, retrasa la nueva acumulación de placa, lo que permite la disminución durante un periodo mayor de tiempo de los niveles de citoquinas en FCG.

En cuanto a los resultados obtenidos tras la aplicación de RAR+TFD, se observó una tendencia a frenar el incremento de los niveles de IL- $1 \beta$ y de TNF- $\alpha$ (principalmente de TNF- $\alpha$ ) en FCG, sin embargo, no concuerdan con los únicos resultados publicados en el literatura sobre el efecto antiinflamatorio de la aplicación de PDT, donde de Oliveira y cols. (32) mostraron una significativa reducción de TNF- $\alpha$ y de RANKL (Receptor del Activador del Factor Nuclear Kappa), aunque no estadísticamente significativa respecto a la obtenida tras el tratamiento con RAR.

La capacidad antioxidante del FCG se ve comprometida en la periodontitis (33). Este compromiso parece deberse más a una consecuencia de la enfermedad inflamatoria que a factores que predisponen a ello
(33). Varios estudios indican que las especies reactivas del oxígeno (ERO) juegan un papel importante en la reacciones fisiológicas e inmunoinflamatorias. En el cuerpo humano hay un mecanismo antioxidante para mantener el equilibrio redox (34). La ruptura de este equilibrio puede conducir a un daño que se ve incrementado por las ERO. De hecho, muchas enfermedades se han correlacionado con la pérdida de este balance redox, o estrés oxidativo. Estudios previos han mostrado que la defensa antioxidante está reducida en la periodontitis crónica, y el tratamiento no quirúrgico con mejoras en los parámetros clínicos puede aumentar dicha defensa antioxidante en los pacientes $(35,36)$. En este estudio, hemos podido mostrar el efecto antiinflamatorio que ejerce la radiación de Er:YAG y cómo el RAR complementado con TFD frena también el proceso inflamatorio, sin embargo, estos hechos no se han traducido en un incremento del estado antioxidante total de FCG.

\section{CONCLUSIONES}

La terapia complementaria con Er:YAG ejerce un efecto antiinflamatorio adicional y, además ralentiza ligeramente el proceso de recolonización.

La terapia complementaria con TFD frena el proceso inflamatorio y ejerce un positivo efecto clínico adicional: una ligera disminución de PSBT.

\section{AGRADECIMIENTOS}

El trabajo descrito en el presente estudio fue financiado por un Proyecto Intramural 200880I215 del Consejo Superior de Investigaciones Científicas, CSIC. Además queremos agradecer a DIEB (Dental IMPLANTS \& BIOMATERIALS, S.L.) por habernos cedido el equipo Periowave y habernos proporcionado Azul de Metileno y a Kavo por prestarnos su equipo Key III Plus.

\section{BIBLIOGRAFÍA}

1. Haper OR, Dymock D, Booth V, Weightman AJ, Wade WG. Detection of unculturable bacteria in periodontal health and disease by PCR. J Clin Microbiol 1999;37:1469-73. 
2. Polson AM, Frederick GT, Ladenheim S, Hanes PJ. The production of a root surface smear layer by instrumentation and its removal by citric acid. J Periodontol 1984;55:443-6.

3. Umeda M, Takeuchi Y, Noguchi K, Huang Y, Koshy G, Ishikawa I. Effects of nonsurgical periodontal therapy on the microbiota. Periodontol $20002004 ; 36: 98-120$.

4. Machion L, Andia DC, Lecio G, Nociti FH Jr, Casati MZ, Sallum AW, Sallum EA. Locally delivered doxycycline as an adjunctive therapy to scaling and root planning in the treatment of smokers: a 2-year follow-up. J Periodontol 2006; 77:606-13.

5. Sharman WM, Allen CM, van Lier JE. Photodynamic therapeutics: basic principles and clinical applications. Drug Discov Today 1999;4: 507-17.

6. Wainwright M, Crossley KB. Photosensitizing agents-circumventing resistance and breaking down biofilms: a review. Int Biodeter Biodegrad 2004;53:119-26.

7. Coffelt DW, Cobb CM, MacNeill S, Rapley JW, Killoy WJ. Determination of energy density threshold for laser ablation of bacteria. An in vitro study. J Clin Periodontol 1997;24:1-7.

8. Gutknecht N, Moritz A, Conrads G, Sievert T, Lampert F. Bactericidal effect of the Nd: YAG laser in in vitro root canals. J Clin Laser Med Surg. 1996;14:77-80.

9. Gutknecht N, Van Gogswaardt D, Conrads G, Apel C, Schubert C, Lampert F. Diode laser radiation and its bactericidal effect in root canal wall dentin. J Clin Laser Med Surg 2000;18:57-60.

10. Schoop U, Moritz A, Kluger W, Patruta S, Goharkhay K, Sperr W, Wernisch J, Gattringer R, Mrass P, Georgopoulos A. The Er: YAG laser in endodontics: results of an in vitro study. Lasers Surg Med 2002;30:360-4.

11. Ishikawa I, Aoki A, Takasaki AA. Potential applications of Erbium: YAG laser in periodontics. J Periodontal Res 2004;39:275-85.
12. Qadri T, Miranda L, Tunér J, Gustafsson A. The short-term effects of low-level lasers as adjunct therapy in the treatment of periodontal inflammation. J Clin Periodontol 2005;32:714-9.

13. Chapple ILC, Landini G, Griffiths GS, Patel NC. Calibration of the Periotron 8000 and 6000 by polinomial regression. J Periodont Res 1999;34: 79-86.

14. Folwaczny M, George G, Thiele L, Mehl A, Hickel R. Root surface roughness following Er:YAG laser irradiation at different radiation energies and working tip angulations. J Clin Periodontol 2002; 29:598-603.

15. Tomasi C, Sahander K, Dahlen G, Wennström JL. Short-Term clinical and microbiologic effects of pocket debridement with Er: YAG laser during periodontal maintenance. J Periodontol 2006; 77:111-8.

16. Jepsen S, Rühling A, König J, Dietzel K, Keller U, Albers HK. Treatment of periodontitis with a novel Er:YAG laser system. J. Dental Res. 2000;79 (Spec Iss): Abstract no. 2281.

17. Christodoulides N, Nikolidakis D, Chondros P, Becker J, Schwarz F, Rossler R, Sculean A. Photodynamic therapy as an adjunct to nonsurgical periodontal treatment: a randomized controlled clinical trial. J Periodontol 2008;79: 1638-44.

18. Chondros P, Nikolidakis D, Christodoulides N, Rössler R, Gutknecht N, Sculean A. Photodynamic therapy as adjunct to non-surgical periodontal treatment in patients on periodontal maintenance: a randomized controlled clinical trials. Lasers Med Sci 2009;24:681-8.

19. Polansky R, Haas M, Heschl A, Wimmer G. Clinical effectiveness of photodynamic therapy in the treatment of periodontitis. J Clin Periodontol 2009;36:575-80.

20. Quirynen M, De Soete $M$, Dierickx K, van Steenberghe D. The intra-oral translocation of periodontopathogens jeopardises the outcome of periodontal therapy. A review of the literature. J Clin Periodontol 2001;28:499-507. 
21. Schwarz F, Sculean A, Georg T, Reich E. Periodontal treatment with an Er:YAG laser compared to scaling and root planing. A controlled study. $\mathrm{J}$ Periodontol 2001;72:361-7.

22. Schwarz F, Sculean A, Berakdar M, Georg T, Reich E, Becker J. Clinical evaluation of an Er:YAG laser combined with scaling and root planing for nonsurgical periodontal treatment. A controlled, prospective clinical study. J Clin Periodontol 2003;30:26-34.

23. Socransky SS, Haffajee AD, Cugini MA, Smit C, Kent RLJr. Microbial complexes in subgingival plaque. J Clin Periodontol 1998;25:134-44.

24. Harper D S, Robinson PJ. Correlation of histometric, microbial, and clinical indicators of periodontal disease status before and after root planing. J Clin Periodontol 1987;14:190-6.

25. Cugini MA, Haffajee AD, Smith C, Kent RLJr, Sokransky SS. The effect of scaling and root planning on the clinical and microbiological parameters of periodontal diseases: 12-month results. J Clin Periodontol 2000;27:30-6.

26. Kolenbrander PE. Oral microbial communities: biofilms, interactions, and genetic systems. Annu Rev Microbiol. 2000;54:413-27.

27. Tsai IS, Tsai CC, Ho YP, Ho KY, Wu YM, Hung CC. Interleukin-12 and interleukin-16 in periodontal disease. Cytokine 2005;31:34-40.

28. Gamanol J, Acevedo A, Bascones A, Jorge O, Silva A. Levels of Interleukin $1 \beta,-8,-10$ and RANTES in gingival crevicula fluid and cell populations in adult periodontitis patients and the effect of periodontal treatment. J Periodontol 2000;71:1535-45.

29. Giannopoulou C, Cappuyns I, Mombelli A. Effect of smoking on gingival crevicular fluid cytokine profile during experimental gingivitis. J Clin Periodontol 2003;30:996-1002.

30. Yoshinari $N$, Kawase $H$, Mitani A, Ito $M$, Sugiishi S, Matsuoka M, Shirozu N, Ishihara Y, Bito B,
Hiraga M, Arakawa K, Noguchi T. Effects of scaling and root planing on the amounts of interleukin-1 and interleukin-1 receptor antagonist and the mRNA expression of interleukin-1beta in gingival crevicular fluid and gingival tissues. J Periodontal Res 2004;39: 158-67.

31. Lopes BM, Marcantonio RA, Thompson GM, Neves LH, Theodoro LH. Short-term clinical and immunologic effects of scaling and root planning with Er:YAG laser in chronic periodontitis. J Periodontol 2008;79:1158-67.

32. de Oliveira RR, Schwartz-Filho HO, Novaes AB, Garlet GP, de Souza RF, Taba M, Scombatti de Souza SL, Ribeiro FJ. Antimicrobial photodynamic therapy in the non-surgical treatment of aggressive periodontitis: cytokine profile in gingival crevicular fluid, preliminary results. J Periodontol 2009;80: 98-105.

33. Chapple ILC, Brock GR, Milward MR, Ling N, Matthews JB. Compromised GCF total antioxidant capacity in periodontitis: cause or effect? J Clin Periodontol 2007;34:103-10.

34. Teng YT. The role of oxygen radicals in human disease with particular acquired immunity and periodontal disease progression. Crit Rev Oral Diseases 2003;6:138-51.

35. Brock GR, Matthews J, Butterwoth C, Chapple ILC. Plasma and crevicular fluid antioxidant defense in periodontitis and health. J Dent Res 2002; 81 (Special Issue A): Abstract 1524.

36. Tsai CC, Chen HS, Chen SL, Ho YP, Ho YM, Hung CC. Lipid peroxidation: a possible role in the induction and progression of chronic periodontitis. J Periodontal Res 2005;40:378-84.

\section{CORRESPONDENCIA}

\section{JA García Núñez}

Dpto. Estomatología III. Facultad de Odontología. Universidad Complutense de Madrid

Plaza Ramón y Cajal, s/n

28040 Madrid, España

garcinu@odon.ucm.es 